\title{
Diversidad de lagartijas en cafetales y bosque primario en el Soconusco chiapaneco
}

\section{Lizard diversity in coffee crops and primary forest in the Soconusco Chiapaneco}

\author{
Rodrigo Macip-Ríos ${ }^{1 *}$ y Antonio Muñoz-Alonso² \\ ${ }^{1}$ Departamento de Zoología, Instituto de Biología, Universidad Nacional Autónoma de México. Apartado postal 70-153, 04510 México, D.F. México \\ ${ }^{2}$ Departamento de Ordenamiento Ecológico, El Colegio de la Frontera Sur, Unidad San Cristóbal de las Casas. Apartado postal 63, 29290 Chiapas, \\ México \\ *Correspondencia: rmr@ibiologia.unam.mx
}

\begin{abstract}
Resumen. La fragmentación y el cambio de uso del suelo hacia actividades primarias intensivas son los factores que tienen más peso en la pérdida de la biodiversidad; no obstante, existen tipos de manejo que pueden tener un equilibrio entre la productividad y la conservación del ambiente; entre ellos se encuentran agrosistemas, como los cafetales, que han sido considerados refugios de la diversidad biológica. En este trabajo se determinó el efecto de estos agrosistemas en la diversidad de lagartijas, encontrándose que es mayor en los cafetales con sombra diversificada de baja altitud que en los sitios de vegetación primaria como la selva mediana y el bosque mesófilo; dicha diversidad se explica porque en los cafetales hay una mayor riqueza de microhábitats disponibles para las lagartijas, los cuales se generan por el tipo de manejo del agrosistema. Este trabajo apoya los cafetales como una opción para la conservación de la biodiversidad; además, se registran observaciones sobre aspectos ecológicos importantes relacionados con las lagartijas, como la apertura y la sobreposición del nicho espacial (microhábitat).
\end{abstract}

Palabras clave: agrosistemas, microhábitat, biodiversidad, refugios, conservación.

\begin{abstract}
Fragmentation and the transformation of natural habitat are the primary factors that promote the loss of biodiversity. However, there are some kinds of land management that may be less aggressive with the environment. Some of these are agrosystems such as coffee crops, which have been considered biodiversity refuges. In this work, we examine lizard diversity in these agrosystems, finding a higher diversity in the low altitude coffee crops than in the primary vegetation such as rain forest, and montane cloud forest. Lizard diversity is explained by the high microhabitat richness in the coffee crops, a pattern also found in birds. This paper supports the idea that the coffee crops work like a refuge for biodiversity and could be useful for conservation biology. Also we report observations of beta diversity and spatial (microhabitat) niche breath and niche overlap.
\end{abstract}

Key words: agrosystems, microhabitat, biodiversity, refuge, conservation.

\section{Introducción}

En las regiones tropicales el cambio de uso de suelo hacia pastizales y monocultivos está considerado una de las principales causas de la pérdida de biodiversidad (Primack, 2002; Urbina y Lodoño, 2003; Gaston y Spicer, 2004). Los cambios estructurales en la vegetación tienen un efecto directo en la composición y abundancia de las especies que forman las comunidades de estos sitios (Heatwole y Taylor, 1987; Pianka, 1994; Lewontin, 1999; Tokeshi, 1999). Por ejemplo, el cambio de selva a potrero genera una gradación de abundancia y diversidad, la cual no necesariamente tiene sus valores más altos en la

Recibido: 06 octubre 2006; aceptado: 03 octubre 2007 vegetación primaria. Éste es el caso de la modificación de la selva alta a pastizal en Los Tuxtlas, Veracruz, México, donde la vegetación de borde representa la mayor riqueza de especies de anfibios y reptiles, seguida por la selva y finalmente el pastizal (Urbina-Cardona y Reynoso, 2005). Urbina-Cardona et al. (2006), mencionan que las características microambientales son determinantes en la composición, abundancia y diversidad de especies de anfibios y reptiles, ya que limitan, o bien, potencializan la presencia de especies en ambientes tropicales. No obstante, la frecuencia e intensidad de las alteraciones antes mencionadas propician efectos diferenciados (Morin, 1999), pues no todas las perturbaciones son necesariamente negativas o generan pérdida de biodiversidad.

Los cafetales son un tipo de uso de suelo muy extendido 
en las zonas montanas tropicales y subtropicales de América (Moguel y Toledo, 1996; Perfecto et al., 1996). El establecimiento de este cultivo implica la transformación del hábitat, aunque la magnitud de ésta depende del modo de cultivo, ya que en algunos, como el rusticano, la transformación estructural y taxonómica de la vegetación, y por lo tanto la conservación de las características físicas y biológicas del hábitat (Moguel y Toledo, 1996); por ello los cafetales se consideran refugios de la biodiversidad (Perfecto et al., 1996; Philpott y Dietsch, 2003). En estos agrosistemas se han registrado una gran cantidad de mamíferos, aves, reptiles e insectos (Greenberg et al., 1997; Rendón et al., 1998; Muñoz et al., 2002; Cruz-Lara et al., 2004).

Los datos anteriores y la gran extensión de terreno en México dedicada al cultivo del café hacen necesario identificar el efecto de éste en la riqueza, abundancia, diversidad y estructura de las comunidades animales que habitan en zonas cafetaleras (Cunninghametal., 2002; Smart et al., 2004). Para ello se utilizó el grupo de las lagartijas, las cuales han sido consideradas como organismos modelo para el estudio de problemas ecológicos (Duellman, 1987; Vitt y Morato, 1995; Vitt y Zani, 1998) por ser animales de captura fácil y sensibles a cambios en la estructura y composición del hábitat, al no poder desplazarse a largas distancias (Smart et al., 2004).

Los objetivos de este trabajo fueron: 1) determinar la riqueza, abundancia y diversidad de especies de lagartijas, tanto en cafetales como en zonas de vegetación primaria y alterada del municipio de Mapastepec, Chiapas, México; 2) identificar el recambio de especies entre los diferentes hábitats (cafetales, vegetación primaria y otro tipo de vegetación perturbada, y 3) determinar la sobreposición del nicho espacial (microhábitat) entre las especies de la zona de estudio para identificar una posible repartición del hábitat.

\section{Materiales y métodos}

El trabajo se realizó en Mapastepec, municipio de la costa de Chiapas, región del Soconusco, ubicado entre $\operatorname{los} 15^{\circ} 40^{\prime} 26^{\prime}$ ' y $15^{\circ} 14^{\prime} 12^{\prime}$ ' $\mathrm{N}$ y $93^{\circ} 06^{\prime} 05^{\prime}$ ' y $92^{\circ} 39^{\prime}$ 12 " O; presenta un intervalo altitudinal de 0 a $2400 \mathrm{~m}$ (INEGI, 2000), donde una gran cantidad de tipos de vegetación tropical (selva alta, selva mediana, vegetación riparia, bosque mesófilo, bosque de encino y en las zonas màs altas bosque de pino) se han visto transformados en los últimos años en pastizales inducidos para el pastoreo, maizales, cacaotales y cafetales de sombra diversificada y de sombra monoespecífica (Inga micheliana).

El clima de Mapastepec es cálido húmedo en el intervalo altitudinal de 0 a $1000 \mathrm{~m}$; aproximadamente de 1000 a $2000 \mathrm{~m}$ cambia hacia semicálido húmedo, y de los $2000 \mathrm{~m}$ en adelante a templado húmedo. Tanto el tipo de clima como la altitud y los tipos de suelo del municipio (regosol, regosol háplico y fluvisol eútrico) lo hacen propicio para las siembra de maíz, café (en las zonas de mediana altitud) y la proliferación de los pastos inducidos para pastoreo en las zonas bajas (INEGI, 1985b; 1985b), lo que propicia que la vegetación primaria haya sufrido una transformación considerable en los últimos años, ya que tanto los pastizales inducidos en las zonas bajas como los cafetales en las estribaciones de la sierra representan el uso de suelo más común.

El trabajo de campo se llevó a cabo en 9 salidas de campo de junio del 2001 a abril del 2002 y las parcelas de muestreo se establecieron en las localidades de Nicolás Bravo II, con una altitud de $350 \mathrm{~m}$ snm y con asociaciones vegetales como pastizal inducido, cafetal de sombra diversificada y relictos de selva alta perennifolia; Unión los Olivos a $700 \mathrm{~m}$ snm con selva mediana subpernnifolia y cafetales de sombra diversificada como vegetación predominante, y Nicolás Bravo I a $1000 \mathrm{~m}$ snm con una vegetación compuesta por cafetales diversificados de sombra monoespecífica y bosque mesófilo de montaña (Muñoz et al., 2002). Nicolás Bravo II se encuentra a 6.12 km de Unión los Olivos, que a su vez se encuentra a 4.26 km de Nicolás Bravo I a vuelo de pájaro. Las características de las parcelas se muestran en el Cuadro1.

En cada parcela se establecieron 3 transectos, los cuales tuvieron una longitud variable (según la extensión de la parcela, con un intervalo de 120-150 m). Estos transectos se establecieron durante la primera salida de campo, en la que también se realizó una cuantificación de los microhábitats que se consideraron como disponibles para las lagartijas de acuerdo con las descripciones de Duellman (1987), Pianka (1993), Canseco (1996) y Vitt et al. (2000): suelo sin vegetación sol/sombra, suelo con vegetación sol/sombra, hojarasca sol/sombra, piedra sol/sombra, tronco caído sol/sombra, tocón sol/sombra, arbusto sol/sombra, grieta, pared de casa, árbol sol/sombra, herbáceas sol/sombra, bromelia.

En este trabajo, la riqueza de microhábitats se considera como una estimación de la heterogeneidad ambiental. Una vez cuantificados los microhábitats se revisaron los transectos una sola vez por salida de campo; en la mañana de 9:00 a 12:00 y en la tarde de 13:00 a 17:00 horas, colectándose todas aquellas lagartijas que se encontraran en el transecto, en una amplitud de $4 \mathrm{~m}$ y a una altura no mayor a $4 \mathrm{~m}$. De cada organismo se anotó: microhábitat, hora del día, actividad, sexo, tipo de parcela y altitud.

Para comprobar que cuando menos se encontraron el $90 \%$ de las especies por parcela, se utilizó un modelo de 
Cuadro 1. Descripción y ubicación de los sitios de muestreo. Las localidades están ordenadas de acuerdo con su disposición altitudninal

\begin{tabular}{|c|c|c|c|c|}
\hline Localidad & $\begin{array}{c}\text { Tipo de vegetación y } \\
\text { clave }\end{array}$ & Características & Altitud & Extensión \\
\hline Nicolás Bravo II & $\begin{array}{l}\text { Cafetal diversificado I } \\
\text { (Cdiv. I) }\end{array}$ & $\begin{array}{l}\text { Ubicado junto a un arroyo, vegeta- } \\
\text { ción original: selva alta; vegetación } \\
\text { circundante, pastizal inducido; pendiente } \\
\text { llana. }\end{array}$ & 300 & 2 ha \\
\hline Nicolás Bravo II & $\begin{array}{l}\text { Cafetal diversificado II } \\
\text { (Cdiv. II) }\end{array}$ & $\begin{array}{l}\text { Ubicado junto a un arroyo; vegetación } \\
\text { original: selva alta, vegetación } \\
\text { circundante: pastizal inducido; pendiente } \\
\text { moderada. }\end{array}$ & 300 & 2 ha \\
\hline Nicolás Bravo II & Pastizal inducido (P) & $\begin{array}{l}\text { Ubicado a } 500 \mathrm{~m} \text { de un cuerpo de agua; } \\
\text { vegetación original: selva alta; vegetación } \\
\text { circundante: cafetales y maizales; } \\
\text { pendiente llana. }\end{array}$ & 300 & 3 ha \\
\hline Unión los Olivos & $\begin{array}{l}\text { Cafetal diversificado III } \\
\text { (Cdiv. III) }\end{array}$ & $\begin{array}{l}\text { Ubicado a } 300 \text { m de un río; vegetación } \\
\text { original: selva mediana; vegetación } \\
\text { circundante: selva mediana y vegetación } \\
\text { secundaria; pendiente moderada }\end{array}$ & 650 & 1 ha \\
\hline Unión los Olivos & $\begin{array}{l}\text { Cafetal diversificado IV } \\
\text { (Cdiv. IV) }\end{array}$ & $\begin{array}{l}\text { Ubicado a } 700 \text { m de un río; vegetación } \\
\text { original: selva mediana; vegetación } \\
\text { circundante: selva mediana y cacaotales, } \\
\text { pendiente moderada }\end{array}$ & 700 & $1.5 \mathrm{ha}$ \\
\hline Unión los Olivos & Selva mediana-alta (SM) & $\begin{array}{l}\text { Ubicada a } 500 \text { m de un cuerpo de } \\
\text { agua; vegetación circundante: cafetales } \\
\text { y vegetación secundaria; pendiente } \\
\text { moderada. }\end{array}$ & 700 & 2.5 ha \\
\hline Nicolás Bravo I & $\begin{array}{l}\text { Cafetal de Inga } \\
\text { micheliana } \\
\text { (C. I. micheliana) }\end{array}$ & $\begin{array}{l}\text { Ubicado a } 1000 \text { m de un río; vegetación } \\
\text { original: bosque mesófilo; vegetación } \\
\text { circundante: vegetación secundaria, } \\
\text { pendiente moderada. }\end{array}$ & 1020 & 3 ha \\
\hline Nicolás Bravo I & $\begin{array}{l}\text { Cafetal diversificado } \mathrm{V} \\
(\text { Cdiv. V) }\end{array}$ & $\begin{array}{l}\text { Ubicado a } 1300 \text { m de un río; vegetación } \\
\text { original: bosque mesófilo; vegetación } \\
\text { circundante: bosque mesófilo., pendiente } \\
\text { moderada. }\end{array}$ & 1050 & 4 ha \\
\hline Nicolás Bravo I & $\begin{array}{l}\text { Bosque } \\
\text { mesófilo (BM) }\end{array}$ & $\begin{array}{l}\text { Ubicado a } 1000 \text { de un río; vegetación } \\
\text { circundante: } \quad \text { cafetales, maizales y } \\
\text { vegetación } \quad \text { secundaria; pendiente } \\
\text { pronunciada. }\end{array}$ & 1070 & 2 ha \\
\hline
\end{tabular}

curvas de acumulación de especies de dependencia lineal, que es el adecuado para áreas pequeñas y para faunas bien conocidas (Soberon y Llorente, 1993). Asimismo, se utilizaron los estimadores no paramétricos Chao 2, ICE y Bootstrap (Urbina-Cardona y Reynoso, 2005). Para estimar la diversidad de especies se usó el índice de Shannon-Wiener (Magurran, 1988; Krebs, 1999); la uniformidad se estimó mediante el índice de equidad de Shannon (Magurran 1988); para determinar diferencias entre la diversidad de los hábitats se llevó a cabo una prueba de $t$ modificada por Hutcheson (Brower et al., 1997; Zar, 1999); para ver si existía una relación entre la diversidad de especies y heterogeneidad ambiental (estimada como riqueza de microhábitats disponibles) se utilizó una regresión lineal simple (Brower et al., 1997; Zar, 1999). Mediante la aproximación de la distribución de Poisson se transformaron los datos de riqueza de microhábitats y de especies, ya que ésta se basó en conteos. Para explorar el recambio de especies se usó el índice de diversidad $\beta$ de Cody (Koleff et al., 2003), y los 
índices de apertura de nicho de Simpson (Vitt y Morato, 1995) y de sobreposición de nicho $\theta j k$ de Pianka (Pianka y Winnemiller,1990) para determinar cómo se dividen el espacio las especies de la zona de estudio. Se realizaron 1000 aleatorizaciones para determinar si el patrón de sobreposición es aleatorio, o bien, es un proceso natural. Para la apertura y sobreposición de nicho únicamente se utilizaron especies con un mínimo de 10 observaciones. Los análisis se llevaron a cabo por medio de los paquetes de computo JMP ver. 5.0.1 (SAS Institute, 2002), Biodiv 5.1 (Baev y Penev, 1996), EstimateS 7 (Colwell, 2004) y EcoSim, ver. 7 (Gotelli y Entsminger, 2001).

\section{Resultados}

Al cabo de 9 salidas de campo se encontraron 16 especies y un total de 443 individuos distribuidos en las 3 localidades muestreadas; éstas se distribuyeron en 5 familias y 10 géneros. La familia de lagartijas con un mayor porcentaje de especies fue la familia Iguanidae (62.50\%), seguida por Teiidae (12.50\%), Scincidae (12.50\%), Geckonidae (6.25\%) y Xantusidae (6.25\%). La abundancia y riqueza encontradas se muestran en el Cuadro 2, donde se observa que los cafetales de baja y media altitud de sombra diversificada (Cdiv I y Cdiv II) fueron aquellos que presentaron una mayor riqueza de especies ( 8 y 7 especies), lo mismo que la selva mediana (7 especies), seguidos por una riqueza intermedia de los Cdiv. IV, Cdiv. V y el pastizal (5 especies); finalmente, el bosque mesófilo y el cafetal de I. micheliana fueron los hábitats con menor riqueza, presentando sólo 4 especies.

En lo que se refiere a riqueza esperada de especies, en todos los hábitats menos en la selva mediana (68.49\% paramétrico y $63 \%$ promedio en los estimadores no paramétricos) se registró arriba del $89 \%$ con el estimador lineal. En el caso de los estimadores no paramétricos los valores fluctuaron de estimador a estimador desde el 64.1 $\%$ hasta el $100 \%$. En la mayoría de los hábitats se encontró más del $80 \%$. El Cuadro 3 muestra los valores de cada estimador.

Los 2 cafetales bajos con sombra diversificada (Cdiv. $\mathrm{I}=1.61$ y Cdiv. II=1.57), la selva mediana (1.55) y el pastizal (1.42) fueron los hábitats con mayor diversidad de especies en forma significativa, aunque sin diferencias entre ellos. En comparación con los cafetales de mediana altitud y sombra diversificada (Cdiv. III=1.07, Cdiv. $\mathrm{V}=1.12$, Cdiv. IV=0.83 ), el cafetal de I. micheliana (1.06) y el bosque mesófilo (1.05) presentaron los valores más bajos de diversidad (Cuadro 4). La diversidad de lagartijas de estos hábitats presentó una relación directa con la riqueza de microhábitats disponibles $\left(\mathrm{r}^{2}=0.6625\right.$ g.1. $=$
$8, \mathrm{p}<0.0076$; diversidad de especies $=0.9242+0.6527$ riqueza de microhábitats; Fig. 1), lo cual indica que a mayor riqueza de microhábitats (entendida como heterogeneidad ambiental), existe una mayor diversidad de especies de lagartijas. La altitud presentó una relación inversa con la diversidad $\left(\mathrm{r}^{2}=0.4759, \mathrm{~g} .1 .=8, \mathrm{P}=0.039\right)$ y riqueza de especies $\left(r^{2}=0.4699\right.$, g. $\left.1 .=8, p=0.041\right)$.

Los valores más altos de diversidad $\beta$ se dieron entre la selva mediana y el cafetal con sombra diversificada de baja altitud I (Cdiv. I), y entre el bosque mesófilo y los cafetales de sombra diversificada de baja altitud (Cdiv.I y Cdiv. II); otros hábitats que presentaron valores altos de diversidad $\beta$ fueron el cafetal de sombra diversificada III y los cafetales de sombra diversificada de baja altitud (Cdiv.I y C.div. II) y el pastizal; el cafetal de sombra de I. micheliana difirió en valores medios de $\beta$ con los otros ambientes (Cuadro 5).

Las especies se observaron en diversos microhábitats y en diferentes frecuencias. Anolis lemurinus se observó en 15 microhábitats, seguida por Ameiva undulata en 13; Anolis sericeus en 12; Basiliscus vittatus en 8; Sceloporus siniferus, Sceloporus teapensis y Anolis matudai en 7; Aspidoscelis deppii en 5; Anolis dollfusianus y Sphenomorphus assatus en 4; Lepidophyma smithi en 3; Corytophanes percarinatus, Hemidactylus frenatus y Mabuya unimarginata en 2, y, finalmente, Anolis leaviventris y Sceloporus ancathinus en 1, aunque en las últimas 5 especies se registraron pocas observaciones.

La apertura del nicho sólo se calculó para 10 especies (con un mínimo de 10 individuos observados) y los valores más altos se obtuvieron para: Anolis lemurinus (0.177), Sceloporus siniferus (0.142) Basiliscus vittatus (0.138) y Anolis sericeus (0.129); mientras que los valores bajos los presentaron: Lepidophyma smithi (0.031), Ameiva undulata (0.040) y Sphenomorphus assatus ( 0.054; Cuadro 6). Es importante destacar que especies como Basiliscus vittatus a pesar de tener un valor alto en la amplitud, es una especie que sólo habita en las orillas de los cuerpos de agua, aunque en ese ambiente (ripario) ocupa una gran cantidad de microhábitats.

En lo referente a la sobreposición del nicho, los análisis se llevaron a cabo con las mismas 10 especies que se utilizaron para calcular la apertura. En este caso los valores de sobreposición se encontraron en un intervalo de 0 y 0.943 (Cuadro 7). Los más altos se presentaron entre Sphenomorpus assatus y A. undulata (0.943); ambas utilizan la hojarasca como microhábitat, pero la primera se desplaza por debajo, mientras que $A$. undulata utiliza la parte superficial; Anolis lemurinus y A. sericeus también presentaron una sobreposición alta (0.767); ambas se encontraron sobre árboles con sombra y sol, pero nunca se les observó en un mismo árbol por 
Cuadro 2. Abundancia por parcela de muestreo de las especies de lagartijas encontradas en el área de estudio, así como la riqueza de especies por parcela de muestreo

\begin{tabular}{|c|c|c|c|c|c|c|c|c|c|c|}
\hline Especies/Parcela de muestreo & $B M$ & $S M$ & $P$ & $\underset{I}{\text { Cdiv. }}$ & $\begin{array}{c}\text { Cdiv. } \\
\text { II }\end{array}$ & $\begin{array}{c}C d v i . \\
I I I\end{array}$ & $\begin{array}{l}\text { Cdiv } \\
I V\end{array}$ & $\begin{array}{c}\mathrm{C} I . \\
\text { micheliana }\end{array}$ & $\underset{V}{C d i .}$ & Total \\
\hline Basilicus vittatus & & & & 5 & 10 & & & & & 15 \\
\hline Corytophanes percarinatus & 1 & 2 & & & & & & & 1 & 4 \\
\hline Hemidactylus frenatus & & 2 & & & & & & & & 2 \\
\hline Sceloporus acanthinus & & & & & & 3 & & & & 3 \\
\hline S. siniferus & & & 7 & 1 & 2 & & & & & 10 \\
\hline S. teapensis & & & & & & & & 19 & 20 & 39 \\
\hline Anolis dollfusianus & 4 & 2 & & & & 4 & 5 & & & 15 \\
\hline A. leaviventris & & & & & & 1 & & & & 1 \\
\hline A. lemurinus & & & 16 & 8 & 15 & & & & & 39 \\
\hline A. matudai & 8 & 4 & & & & 2 & 3 & 3 & 4 & 24 \\
\hline A. sericeus & & 1 & 10 & 23 & 9 & 6 & 3 & & 1 & 53 \\
\hline Ameiva undulata & 1 & 14 & 3 & 27 & 42 & 45 & 40 & 17 & 16 & 205 \\
\hline Aspidoscelis deppii & & 3 & 3 & & & & & & & 6 \\
\hline Mabuya unimarginata & & & & 2 & & & & & & 2 \\
\hline Sphenomorphus assatus & & & & 2 & 5 & 2 & 1 & 2 & & 12 \\
\hline Lepidophyma smithi & & & & 6 & 7 & & & & & 13 \\
\hline Total & 14 & 28 & 39 & 74 & 90 & 63 & 52 & 41 & 42 & 443 \\
\hline Riqueza & 4 & 7 & 5 & 8 & 7 & 7 & 5 & 4 & 5 & $16 / 443$ \\
\hline
\end{tabular}

BM: Bosque mesófilo, SM: Selva mediana, Cdiv. I: Cafetal diversificado I, Cdiv. II: Cafetal diversificado II, Cdiv. III: Cafetal diversificado III, C I. micheliana: Cafetal de Inga micheliana, Cdiv. IV: Cafetal diversificado IV, Cdiv V: Cafetal diversificado V. En el extremo inferior derecho se muestra el número total de especies sobre el número total de individuos.

Cuadro 3. Estimadores no paramétricos y paramétricos (modelo lineal) de la riqueza esperada. Los valores entre paréntesis representan el porcentaje de completness de cada hábitat. $S_{o b s}=$ riqueza observadad, $S=$ Singlentons, $D=$ Doubletons

\begin{tabular}{lccccccc}
\hline \multicolumn{1}{c}{ Hábitat } & $S_{\text {obs }}$ & Chao2 & ICE & Bootstrap & Modelo lineal & $S$ & $D$ \\
\hline BM & 4 & $3.5(100)$ & $6.24(64.10)$ & $4.78(83.68)$ & $3.97(100)$ & 2 & 0 \\
SM & 7 & $11(63.63)$ & $14.91(46.94)$ & $8.71(80.36)$ & $10.22(68.49)$ & 1 & 3 \\
P & 5 & $5.18(96.52)$ & $5.38(92.93)$ & $5.51(90.74)$ & $5.15(97.08)$ & 0 & 0 \\
Cdiv. I & 8 & $8.25(96.96)$ & $8.43(94.89)$ & $8.76(91.32)$ & $8.46(94.56)$ & 1 & 2 \\
Cdiv. II & 7 & $6(100)$ & $7.31(95.75)$ & $7.42(94.33)$ & $6.95(100)$ & 0 & 1 \\
Cdiv. III & 7 & $7.66(91.38)$ & $8.91(78.56)$ & $8.11(86.31)$ & $7.82(89.51)$ & 1 & 2 \\
Cdiv. IV & 5 & $5(100)$ & $5(100)$ & $5.34(93.63)$ & $5.36(93.28)$ & 0 & 0 \\
C. I. micheliana & 4 & $4(100)$ & $4(100)$ & $4.30(93.02)$ & $4.43(90.29)$ & 0 & 1 \\
Cdiv V & 5 & $7(71.42)$ & $6.91(72.35)$ & $5.87(85.17)$ & $5.51(90.71)$ & 1 & 1 \\
\hline
\end{tabular}

BM: Bosque mesófilo, SM: Selva mediana, P: Pastizal. Cdiv. I: Cafetal diversificado I, Cdiv. II: Cafetal diversificado II, Cdiv. III: Cafetal diversificado III, Cdiv. IV: Cafetal diversificado IV, C I. micheliana: cafetal de Inga micheliana, Cdiv. V: cafetal diversificado $\mathrm{V}$. Las letras a y b en la $t$ de Hutchenson indican las diferencias entre los hábitats. 
Cuadro 4. Diversidad y equitatividad en las parcelas de muestreo

\begin{tabular}{|c|c|c|c|c|c|c|c|c|c|}
\hline & $B M$ & $S M$ & $P$ & Cdiv.I & $\begin{array}{c}\text { Cdiv. } \\
\text { II }\end{array}$ & $\begin{array}{c}\text { Cdiv. } \\
\text { III }\end{array}$ & $\begin{array}{c}\text { Cdiv. } \\
I V\end{array}$ & $\begin{array}{c}\text { C. I. } \\
\text { micheliana }\end{array}$ & $\begin{array}{c}\text { Cdiv. } \\
\quad V\end{array}$ \\
\hline Diversidad (Shannon) & 1.05 & 1.55 & 1.42 & 1.61 & 1.57 & 1.07 & 0.83 & 1.06 & 1.12 \\
\hline Equitatividad (Shannon) & 0.76 & 0.79 & 0.88 & 0.77 & 0.80 & 0.54 & 0.51 & 0.76 & 0.69 \\
\hline$t$ de Hutchenson $(\mathrm{P}<0.05)$ & $\mathrm{b}$ & a & a & $\mathrm{a}$ & $\mathrm{a}$ & $\mathrm{b}$ & $\mathrm{b}$ & $\mathrm{b}$ & $\mathrm{b}$ \\
\hline
\end{tabular}

BM: Bosque mesófilo, SM: Selva mediana, P: Pastizal. Cdiv. I: Cafetal diversificado I, Cdiv. II: Cafetal Inga micheliana, Cdiv. V: cafetal diversificado V. Las letras a y b en la $t$ de Hutchenson indican las diferencias entre los hábitats.

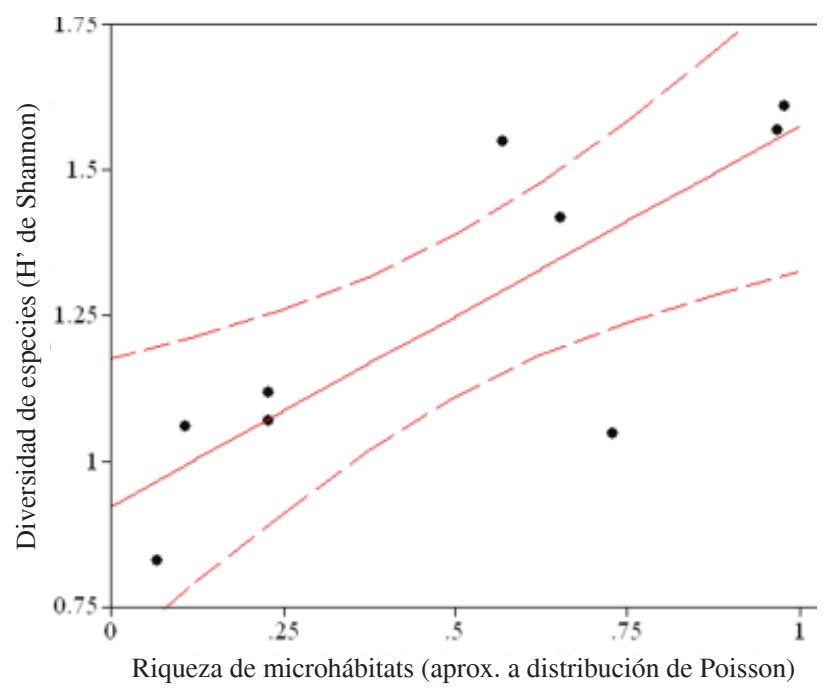

Figura 1. Relación entre la diversidad de especies de lagartijas y la riqueza de microhábitats en los diferentes cafetales y el bosque primario.

grande que este fuera; asimismo, Anolis matudai y $A$. dollfusianus presentaron una sobreposición considerable (0.675), fueron observadas en cafetos y en la hojarasca, pero A. matudai habita en sitios más asociados al suelo, como troncos caídos y hojarasca; A. dollfusianus, en cafetos y ramas de arbustos; A. dollfusianus y Sceloporus teapensis (0.546) presentaron una sobreposición media, pues $S$. teapensis ocupa microhábitats soleados y a orilla del bosque o cafetal, mientras que $A$. dollfusianus es una especie del interior del bosque, aunque algunas veces se encuentra en la orilla, donde la insolación es mayor. El patrón de sobreposición encontrado en esta comunidad de lagartijas no fue aleatorio, es decir, lo observado responde a procesos naturales como la repartición de recursos y no la competencia. La media observada $(0.180)$ fue diferente de manera significativa de la media ponderada $(0.126)$ en 1000 aleatorizaciones $(\mathrm{P}=0.042)$.

La sobreposición fue media o media baja en pares como: Anolis sericeus con Sceloporus siniferus (0.445) y A. lemurinus con $S$. siniferus (0.310). A estos valores medios le siguen la mayoría de los resultados de sobreposición, que en su mayoría son bajos (Cuadro7). En términos generales, la sobreposición del nicho espacial (microhábitat) en el ensamble de especies es baja, pues sólo pocas especies presentan similitud en los microhábitats que utilizan.

\section{Discusión}

Los datos sugieren que la mayor diversidad ecológica observada en los diferentes hábitats muestreados está dada por la cantidad de microhábitats disponibles para lagartijas en esos ambientes, es decir, por la heterogeneidad ambiental (para las lagartijas), como ya fue observado en lagartijas del desierto (Pianka, 1966, 1976 y 1994), de bosques tropicales (Vitt y Morato, 1995) y asociadas a hábitats riparios (Jones, 1981). Sin embargo, el bosque mesófilo, a pesar de ser un hábitat muy heterogéneo, presentó un valor bajo en la diversidad de especies, lo que se debe a que es un hábitat poco adecuado para las lagartijas, ya que el clima templado, la poca iluminación y la alta humedad limitan la presencia de especies con complejo comportamiento termorregulador y sólo lo habitan aquellas consideradas como termoconformistas (Pianka, 1993).

En cacaotales (agrosistemas similares a los cafetales) también se ha encontrado una alta diversidad ecológica de anfibios y reptiles de la hojarasca (Heinen, 1992), fenómeno atribuido a la complejidad del hábitat y a cierta similitud estructural con la vegetación primaria. Específicamente en cafetales se ha encontrado este mismo patrón en anfibios (Pineda y Halffter, 2003), reptiles (Percino, 2001), aves (Greenberg et al., 1997) y mamíferos (Ramírez-Lozano, 
Cuadro 5. Diversidad $\beta$ de Cody de los hábitats muestreados

\begin{tabular}{|c|c|c|c|c|c|c|c|c|c|}
\hline & Cdiv. I & Cdiv. II & $P$ & Cdiv. III & $S M$ & Cdiv. IV & $\begin{array}{c}C I . \\
\text { micheliana }\end{array}$ & Cdiv. V & $B M$ \\
\hline Cdiv. I & _ & 0.5 & 2.5 & 4 & 5.5 & 2.5 & 3.5 & 3 & 5 \\
\hline Cdiv. II & & - & 2 & 4 & 5 & 2.5 & 3.5 & 4 & 5 \\
\hline $\mathrm{P}$ & & & _ & 4 & 3 & 2.5 & 3.5 & 3 & 3.5 \\
\hline Cdiv. III & & & & - & 3 & 1 & 2.5 & 3 & 1 \\
\hline SM & & & & & _ & 2.5 & 3.5 & 2 & 1.5 \\
\hline Cdiv. IV & & & & & & - & 1 & 1.5 & 2 \\
\hline CInga. & & & & & & & - & 1.5 & 2 \\
\hline Cdiv. V & & & & & & & & - & 1.5 \\
\hline $\mathrm{BM}$ & & & & & & & & & _ \\
\hline
\end{tabular}

Cuadro 6. Apertura de nicho de las 10 especies de lagartijas mejor representadas en distintos tipos de cafetal y bosque primario

\begin{tabular}{lccc}
\hline \multicolumn{1}{c}{ Especie } & Núm.. de microhábitats & $N$ & Apertura de nicho (0-1) \\
\hline Anolis lemurinus & 15 & 39 & 0.177 \\
Sceloporus siniferus & 7 & 10 & 0.142 \\
Basilicus vittatus & 8 & 15 & 0.138 \\
Anolis sericeus & 12 & 53 & 0.129 \\
A. dollfusianus & 4 & 14 & 0.113 \\
A. matudai & 7 & 24 & 0.108 \\
Sceloporus teapensis & 7 & 39 & 0.064 \\
Sphenomorphus assatus & 4 & 12 & 0.054 \\
Ameiva undulata & 13 & 205 & 0.040 \\
Lepidophyma smithi & 3 & 13 & 0.031 \\
\hline
\end{tabular}

2002; Cruz-Lara, 2004). En una zona cercana al área de estudio, Percino (2001) menciona una mayor riqueza y diversidad de especies de reptiles en los cafetales, que atribuye a un incremento de especies oportunistas por la transformación del ambiente.

En este trabajo, la altitud fue otro factor que tiene influencia en la diversidad de especies, pues las parcelas a más baja altitud fueron las que presentaron los valores más altos de riqueza y diversidad. Este patrón de diversidad es bien conocido en varios grupos de vertebrados, entre otros los reptiles (Morin, 1999). La teoría del disturbio intermedio propuesta por Connell (1978) determina que en un ambiente con mediana perturbación y en lapsos medios. la presencia de especies será mayor que en la de un hábitat conservado o en la de uno sujeto a perturbación intensa, pues en ambientes "intermedios" pueden coexistir especies de zonas prístinas con las de zonas perturbadas (Morin, 1999), tal como encontró Urbina-Cardona y Reynoso (2005) en Los Tuxtlas en una matriz de potreroborde-selva.

Los elevados valores de diversidad $\beta$ entre las parcelas de vegetación primaria, como la selva mediana y el bosque mesófilo, con la vegetación transformada como los cafetales de baja altitud, parecen explicarse por una tendencia de los organismos terrestres medianos y pequeños a presentar valores altos en la diversidad $\beta$ (Arita, 1997) y no necesariamente sugieren faunas distintas o una degradación de los hábitat primarios. Respecto a los mamíferos pequeños no voladores y roedores, Arita (1997) y Ramírez-Lozano (2002) argumentan que los valores altos de diversidad $\beta$ se deben a su reducida capacidad de dispersión (el caso de las lagartijas) en ambientes topográfica y ecológicamente 
Cuadro 7. Matriz de sobreposición del nicho espacial (microhábitat) para 10 especies de lagartijas encontradas en distintos tipos de cafetal y en bosque primario

\begin{tabular}{|c|c|c|c|c|c|c|c|c|c|c|}
\hline & Bas v & Sces & Scet & And & An le & An $m$ & An se & $A m u$ & Sph a & Leps \\
\hline $\operatorname{Bas} v$ & 1 & 0.041 & 0.021 & 0 & 0.192 & 0.014 & 0.139 & 0.007 & 0 & 0 \\
\hline Sces & & 1 & 0.118 & 0.037 & 0.310 & 0 & 0.445 & 0.249 & 0.231 & 0 \\
\hline Sce $t$ & & & 1 & 0.546 & 0.219 & 0.191 & 0.184 & 0.295 & 0.279 & 0.011 \\
\hline And & & & & 1 & 0.339 & 0.675 & 0.212 & 0.275 & 0.414 & 0.053 \\
\hline An le & & & & & 1 & 0.287 & 0.767 & 0.169 & 0.234 & 0.024 \\
\hline$A n m$ & & & & & & 1 & 0.302 & 0.142 & 0.154 & 0.008 \\
\hline An se & & & & & & & 1 & 0.028 & 0.034 & 0.004 \\
\hline$A m u$ & & & & & & & & 1 & 0.943 & 0.001 \\
\hline Sph a & & & & & & & & & 1 & 0.027 \\
\hline Lep s & & & & & & & & & & 1 \\
\hline
\end{tabular}

Bas v: Basiliscus vittatus; Sce s: Sceloporus siniferus; Sce t: Sceloporus teapensis; An d: Anolis dollfusianus; An le: Anolis lemurinus; An m: Anolis matudai; An se: Anolis sericeus; Am u: Ameiva undulata; Sph a: Sphenomorphus assatus; Lep s: Lepidophyma smithi.

heterogéneos (como la matriz de cafetales, vegetación primaria, bordes y pastizales). Esto incrementa de forma importante la diversidad regional de un área, lo cual se considera uno de los principales promotores de que grupos como los de reptiles o mamíferos pequeños sean faunas tan diversas en países con las características topográficas y climáticas de México.

Los resultados de este trabajo indican que algunos cafetales con características especiales, como estar ubicados a baja altitud y ser del tipo rusticano, fungen como un refugio para la biodiversidad, tal como lo han propuesto Mogel y Toledo (1996), Perfecto et al. (1996) y Philpott y Dietsch (2003), pese a que existen argumentos en contra, como la pérdida de especies exclusivas de los hábitat primarios, la poca productividad de los cafetales diversificados comparados con los de sol y una ambigua forma de certificación del café como producto amigable de la biodiversidad (Rappole et al., 2003a; Rappole et al., 2003b). Consideramos, que los cafetales son una opción adecuada para la conservación en un contexto donde lo imperativo es la transformación de las áreas silvestres hacia pastizales, ambientes que como lo indican los datos que se registran en este trabajo tienen una baja diversidad ecológica y una pobre heterogeneidad ambiental (incluida la completa pérdida de la cobertura arbórea).

Tanto la heterogeneidad ambiental como la altitud presentan un efecto aditivo en la riqueza y diversidad de especies. Sin embargo, el diseño del estudio no fue simétrico, es decir, no existen replicas del pastizal en los 2 pisos altitudinales superiores a los $350 \mathrm{~m}$, ni del bosque mesófilo por debajo de los $1000 \mathrm{~m}$, ni de un hábitat conservado a baja altitud. En los trabajos de campo es difícil encontrar sistemas balanceados y más en una zona con una transformación histórica del paisaje tan compleja como el Soconusco chiapaneco. Los efectos confundidos derivados del diseño se limitan únicamente a la heterogeneidad ambiental y la altitud, componentes fundamentales en la diversidad local de especies (Morin, 1999). El análisis de la dimensión espacio (microhábitat) que se menciona en los párrafos siguientes, esclarece esta confusión de manera importante, pues los datos muestran que en los ensambles de especies estudiados, el nicho espacial es fundamental para la estructuración de las comunidades de lagartijas del sitio de estudio.

Respecto a la apertura del nicho espacial (microhábitat) se encontró un patrón predecible: pocas especies generalistas y muchas especialistas (Pianka, 1994), pese a que la teoría de la biología de conservación menciona que los organismos especialistas son más sensibles a la transformación del hábitat (Primack, 2002). En este caso, una especie en particular se ha visto beneficiada con el cambio de vegetación primaria a cafetal. Ameiva undulata, típica de la orilla del bosque, incrementó su abundancia relativa en las parcelas del cafetal; un caso particularmente interesante, pues como se observa en el Cuadro 2 muestra una abundancia relativa que cuadriplica la de las zonas de vegetación primaria; sin embargo, en el pastizal, donde la insolación es muy alta, tiene una abundancia limitada. 
En algunas zonas, como Los Tuxtlas, A undulata entra en los potreros pero no en zonas conservadas (UrbinaCardona et al., 2006). Esta especie ha encontrado en los cafetales condiciones microambientales muy favorables, como una insolación media, gran cantidad de hojarasca (el microhábitat que más utiliza) y humedad adecuada. Para A. undulata los cafetales funcionan como un gran borde, de ahí su éxito en la zona de estudio, que explica la gran abundancia de una especie con un nicho reducido aunque subsidiado, en este caso particular. En un estudio en el Cerrado, Brasil, Gainsbury y Colli (2003) encontraron un patrón similar con Ameiva ameiva, la cual se encuentra en todos los sitios que muestrearon en abundancias muy altas.

Lepidophyma smithi, otra especie considerada como especialista, sólo se encontró en los cafetales de baja altitud, donde el microhábitat que más utiliza (troncos podridos húmedos) se encuentra presente en grandes números. Esta especie tiene requerimientos muy especializados en cuanto al hábitat y al microhábitat, ya que requiere de mucha humedad y sombra para evitar la elevada evapotranspiración que la caracteriza (Mautz, 1982). En este caso los cafetales han fungido como un refugio, pues en una matriz de pastizales y maizales, fuera de la vegetación primaria (la cual es escasa), los cafetales diversificados son los únicos ambientes que puden albergar estas especies. Su ausencia del bosque mesófilo responde a una distribución altitudinal restringida a zonas bajas. Phrynosomatidos, como las especies del género Sceloporus, Polychrotidos, como las de Anolis, e incluso Corithophanidos, como Basiliscus vittatus presentaron valores altos de amplitud de nicho espacial. Especies de este grupo explotan por lo general una mayor cantidad de recursos, aunque $B$. vittatus está restringida a los ambientes riparios. Los datos de amplitud del nicho ecológico indican cómo está repartida la dimensión espacio en la zona de estudio; pocas con amplitud elevada y numerosas con amplitud restringida.

Cuando la sobreposición es baja (en este caso el microhábitat) en una de las dimensiones del nicho, ésa tiene el papel más importante en el ensamble de la comunidad (Pianka, 1986; Ortega et al., 1992), lo cual es similar a los resultados de este trabajo. Esto se ha encontrado ya en comunidades de lagartijas tropicales (Duellman, 1987; Vitt y Zani, 1998; Vitt et al., 2000) y en zonas áridas rocosas (Howard y Hailey, 1999).

Aunque no se llevó a cabo el análisis de la dimensión tiempo y dieta, la primera sólo tiene peso cuando existen muchas especies nocturnas (Pianka y Pianka, 1976) y en el caso de la dieta, indirectamente es posible inferir que la sobreposición en esta dimensión es alta, pues la teoría del nicho predice que si la sobreposición es baja en alguna dimensión en otra es alta (Pianka, 1986). Por otra parte
Howard y Hailey (1999) mencionan que en los ambientes homogéneos las comunidades se estructuran por el alimento, ya que al existir pocos microhábitats, éstos son ocupados por completo, al contrario de lo que sucede en ambientes heterogéneos donde las comunidades se estructuran por la dimensión espacio (microhábitat), el cual es más diverso, lo que parece suceder en la comunidad de lagartijas que aquí se estudiaron.

En el escenario del corredor biológico mesoamericano, un gran esfuerzo de conservación que pretende unir áreas naturales desde Panamá hasta el sureste mexicano (que incluye el área de estudio en la zona que comprende el corredor entre las reservas de la biosfera El Triunfo y La Sepultura), se vuelve necesario encontrar estrategias que además de ser productivas y sustentables para los habitantes de la zona permitan el flujo de individuos entre hábitats, es decir, que se recupere la cobertura vegetal y se haga un uso sustentable del ambiente. Autores como Bartra (2001) vislumbran el cultivo del café como una de las alternativas más fuertes para el desarrollo regional en mesoámerica. Los datos de este trabajo, desde el punto de vista de la diversidad de lagartijas, apoyan la hipótesis de que algunos tipos de este agrosistema, como los cafetales de sombra diversificada (rusticano) en altitudes menores a los $700 \mathrm{~m}$ snm, funcionan como un refugio para la biodiversidad y plantean una estrategia adecuada para su conservación.

Es también necesario mencionar que algunos datos aquí presentados (la menor riqueza y diversidad de lagartijas en los bosques mesófilos de montaña en comparación con otros hábitat, como el pastizal de baja altitud) no se interpretan de manera absoluta. Ya otros trabajos (Percino, 2001) han encontrado menor diversidad de reptiles en estos ambientes; no obstante, los bosques mesófilos son ricos en endemismos y con flora y fauna únicas, además tienen un valor biológico y funcional fundamental por los servicios ambientales que proveen. Los datos del trabajo simplemente reflejan que son un hábitat que limita la distribución de algunos reptiles con complejos modos de regulación de la temperatura.

\section{Agradecimientos}

Al Instituto para el Desarrollo Sustentable de Mesoamérica, A.C. (IDESMAC) y al Banco Mundial por el apoyo financiero para la realización de este trabajo. RMR agradece al Colegio de la Frontera Sur, Unidad San Cristóbal, a Mariana Ramírez, Marilú Moreno y Pilar Martínez por su apoyo en el trabajo de campo, así como al Dr. Gustavo Casas Andreu quien realizo valiosos comentarios a este manuscrito. Dos revisores anónimos 
contribuyeron a mejorar este escrito.

\section{Literatura citada}

Arita, H. 1997. The non-volant mammal fauna of México: species richness in a megadiverse country. Biodiversity and Conservation 6:787-795.

Baev, P. y L. Penev. 1996. Biodiv 5.1. Pensoft, Sofia 50 p.

Bartra, A. 2001. Sur. Megaplanes y utopías en la América equinoccial. In Mesoamérica. Los ríos profundos. A. Bartra (ed.). Instituto Maya; El Atajo; Fomento Cultural y Educativo, México, D.F. p. 13-132.

Brower, J., J. Zar y C. von Ende. 1997. Field and laboratory methods of general ecology, cuarta edición. McGraw-Hill, Boston, Massachusetts. 273 p.

Canseco, L. 1996. Estudio preliminar de la herpetofauna de la cañada de Cuicatlán y cerro Piedra Larga, Oaxaca. Tesis, Escuela de Biología, Benemérita Universidad Autónoma de Puebla. México. 180 p.

Colwell, R. K. 2004. EstimateS 7: Statistical estimation of species richness and shared species from samples. University of Conneticut, Storrs.

Connell, J. H. 1978. Diversity in tropical rain forest and coral reefs. Science 199:1302-1310.

Cunningham, S. C., R. D. Babb, T. R. Jones, B. D. Taubert y R. Vega. 2002. Reaction of lizard populations to a catastrophic wildfire in a central Arizona mountain range. Biological Conservation 107:193-201.

Cruz-Lara, L. C. Lorenzo, L. Soto, E. Naranjo y N. RamírezMarcial. 2004. Diversidad de mamíferos en cafetales y selva mediana de las cañadas de la selva lacandona, Chiapas, México. Acta Zoológica Mexicana 20:63-81.

Duellman, E. W. 1987. Lizards in an Amazonian rain forest community resource utilization and abundance. National Geographic Research 3:489-500.

Gainsbury, A. M. y G. R. Colli. 2003. Lizard assemblages from natural cerrado enclaves in southwestern Amazonia: the role of stochastic extinctions and isolation. Biotropica 35:503519.

Gaston, K. J. y J. I. Spicer. 2004. Biodiversity. An introduction, segunda edición. Blackwell Science, Malden, Massachusets. $191 \mathrm{p}$.

Gotelli, N. J. y G. L. Entsminger. 2001. EcoSim: Null Models software for ecology. Ver. 7.0.Acquired Intelligence \& Kesey-Bear.http://homepages.together.net/gentsmin/ecosim. htm

Greenberg, R., P. Bichier, A. Cruz y R. Reitsma. 1997. Bird populations in shade and sun coffee plantations in Central America. Conservation Biology 11:448-459.

Heatwole, H. y J. Taylor. 1987. Ecology of reptiles. Surreay Beatty, Melburn, Australia. 325 p.

Heinen, T. J. 1992. Comparisons of the leaflitter herpetofauna in abandoned cacao plantations and primary rain forest in Costa Rica: some implications for faunal restoration. Biotropica 24:431-439.

Howard, K. y A. Hailey. 1999. Microhabitat separation among diurnal saxicolous lizards in Zimbabwe. Journal of Tropical Ecology 15:367-378.
INEGI (Instituto Nacional de Estadística, Geografía e Informática). 1985a. Carta de uso de suelo y vegetación. Huixtla (D15-2). 1:250000. México.

INEGI (Instituto Nacional de Estadística, Geografía e Informática). 1985b. Carta edafológica. Huixtla (D15-2) 1:250000. México, D.F.

INEGI (Instituto Nacional de Estadística, Geografía e Informática). 2000. Anuario estadístico de Chiapas. INEGIGobierno del Estado de Chiapas, Tuxtla Gutiérrez, 506 p.

Jones, B. K. 1981. Efects of grazing on lizard abundance and diversity in western Arizona. The Southwestern Naturalist 26:107-105.

Koleff, P., K. J. Gaston, J. y J. Lennon. 2003. Measuring beta diversity for presence-absence data. Journal of Animal Ecology 72:367-382.

Krebs, C. 1999. Ecological methodology, segunda edición, Addison Wesley, Menlo Park, California. 620 p.

Lewontin, R. 1999. La evolución. 25-33. In La evolución biológica, J. Núñez-Farfán y L. Eguiarte (eds.). Universidad Nacional Autónoma de México y Comisión Nacional para el Conocimiento y Uso de la Biodiversiad, México, D.F. 457 p.

Magurran, A. 1988. Ecological diversity and its measurement. Princeton University Press, New Jersey. 179 p.

Mautz, W. 1982. Use of cave resources by a lizard community. In Herpetological Communities: A symposium of the Society for the Study of Anphibians and Reptiles and The Herpetologists League, August 1977. N. Scott (ed.). Wildlife Research Report 13. U. S. Fish and Wildlife Service, Washington, D.C. p. 129-134.

Moguel, P. y V. Toledo. 1996. El café en México, ecología, cultura indígena y sustentabilidad. Ciencias 43:40-51.

Morin P. J. 1999. Community Ecology. Blackwell Science, Malden, Massachusets. 423 p.

Muñoz, A., A. Horváth, R. Percino, R. Ramírez, R. Macip, R. Martínez, M. Moreno, M. Ramírez y R. Vidal. 2002. Evaluación de la diversidad de vertebrados terrestres en cafetales de la Reserva de la Biosfera El Triunfo. Informe final. El Colegio de la Frontera Sur (ECOSUR-IDSMAC). Chiapas. 67 p.

Ortega, A., R. Barbault y A. Romero. 1992. The ecological organization of a highly diverse desert lizard community in Mexico. In Vertebrate ecology in arid zones of Mexico and Asia, V. Sokolov, G. Halffter y A. Ortega (eds.). Instituto de Ecología, Centro de Investigaciones Biológicas de Baja California Sur, Hombre y Biosfera (MAB, por sus siglas en inglés) -UNESCO), México. p. 207-238.

Percino, R. 2001. Diversidad de reptiles en bosque mesófilo de montaña y cafetal, en la Reserva de la Biosfera El Triunfo, Chiapas, México. Tesis, Benemérita Universidad Autónoma de Puebla, Puebla. 94 p.

Perfecto, I., R. Rice, R. Greenberg y M. Van der Voort. 1996. Shade coffee: a disapearing refuge for biodiversity. BioScience 46:598-608.

Philpott, S. M., y T. Dietsch. 2003. Coffee and conservation: a global context and the value of farmer involment. Conservation Biology 17:1844-1846.

Pianka, E. 1966. Convexity, desert lizards and spatial heterogeneity. Ecology 47:1055-1059. 
Pianka, E. 1976. Lizard species diversity. Ecology 48:333-351.

Pianka, E. 1986. Ecology and natural history of desert lizards. Princeton University Press, New Yersey. 208 p.

Pianka, E. 1993. The many dimensions of the lizard ecological niche. In Lacertids of the Mediterranean region. A biological approach, E. Valakos, W. Böhem, V. Pérez y P. Maragou. (eds.). Hellenic Zoological Society, Athens. p. 121-154.

Pianka, E. 1994. Biodiversity of australian desert lizards. In Biodiversity and terrestrial ecosystems, Monographs Series 14, C. Peng y C. H. Chou (eds.). Institute of Botany, Academia Sinica, Taipei. p. 259-281.

Pianka, E. y H. Pianka. 1976. Comparative ecology of twelve species of nocturnal lizards (Gekkonidae) in Western Australia desert. Copeia 1976:125-142.

Pianka, E. y K. Winemiller. 1990. Organization in natural assemblages of desert lizards and tropical fishes. Ecological Monographs 60:27-55.

Pineda, E., y G. Halffter. 2003. Species diversity and habitat fragmentation: frogs in a tropical montane landascape in México. Biological Conservation 117:499-408.

Primack, R. 2002. Essentials of Conservation Biology, tercera edición, Sinauer, Sunderland, Massachusetts. 698 p.

Ramírez-Lozano, M. 2002. Diversidad de roedores en cafetales de la Reserva de la Biosfera "El Triunfo", Chiapas, México. Tesis, Escuela de Biología, Benemérita Universidad Autónoma de Puebla, Puebla. 66 p.

Rappole, J. H., D. I. King y J. H. Vega-Rivera. 2003a. Coffee and conservation. Conservation Biology 17:334-336.

Rappole, J. H., D. I. King y J. H. Vega-Rivera. 2003b. Coffee and conservation III: A reply to Philpott and Dietsch. Conservation Biology 17:1847-1849.

Rendón, A., T. Álvarez y O. Flores-Villela. 1998. Herpetofauna de Santiago Jalahui, Oaxaca, México. Acta Zoológica Mexicana 75:17-45.

SAS Institute. 2002. JMP. Statistical Discovery Software. Ver. 5.0.1. Cary, North Carolina.

Soberón, J. y J. Llorente. 1993. The use of species acumulation functions for the predictions of species richness. Conservation
Biology 7:480-488.

Smart, R., M. J. Whiting y W. Twine. 2002. Lizards and landscapes: integrating field surveys and interviews to asses the impact of human disturbance on lizard assemblages and selected reptiles in a sabana in South Africa. Biological Conservation 122:23-31.

Tokeshi, M. 1999. Species coexistence. Ecological and evolutionary perspectives. Blackwell Science, Osney Mead. $454 \mathrm{p}$.

Urbina, J. N. y M. C. Lodoño. 2003. Distribución de la comunidad de herpetofauna asociada a cuatro áreas con diferente grado de perturbación en la Isla Gorgona, Pacífico colombiano. Revista de la Academia Colombiana de Ciencia 27:105113.

Urbina-Cardona, J. N. y Reynoso, V. H. 2005. Recambio de anfibios y reptiles en el gradiente potrero-borde-interior de la selva en la Reserva de Los Tuxtlas, Veracruz, México. In Sobre diversidad biológica: el significado de las diversidades alfa, beta y gamma, G. Halffter, J. Soberón, P. Koleff y A. Melic (eds.). Comisión Nacional para el Conocimiento y Uso de la Biodiversidad, Sea, Diversitas, Consejo Nacional de Ciencia y Tecnología. Zaragoza. p. 191-208.

Urbina-Cardon, J. N., M. Olivares-Pérez y V. H. Reynoso. 2006. Herpetofauna diversity and microcroenviroment correlates across a pasture-edge-interior ecotone in tropical rainforest fragments in the Los Tuxtlas Biosphere Reserve of Veracruz, Mexico. Biological Conservation 132:61-75.

Vitt, L. y C. Morato. 1995. Niche partitioning in a tropical wet season: lizards in the Lavrado area of northern Brazil. Copeia 1995:305-329.

Vitt, L. y P. Zani. 1998. Ecological relationships among sympatric lizards in a transittional forest in the northern Amazon of Brazil. Journal of Tropical Ecology 14:63-86.

Vitt, L., S. Sartorius, S. Ávila, Espósito y B. Miles. 2000b. Niche segregation among sympatric amazonian teid lizards. Oecologia 122:410-420.

Zar, J. H. 1999. Biostatistical Analysis, cuarta edición. Prentice Hall, New Jersey. 662 p. 